\title{
Cannabis e Câncer de mama: Evidências com células in vitro
}

\author{
Cannabis and Breast Cancer: Evidence with In Vitro Cells \\ Cannabis y cáncer de mama: Evidencia con células in vitro
}

Recebido: 18/07/2021 | Revisado: 25/07/2021 | Aceito: 27/07/2021 | Publicado: 04/08/2021

\author{
Milena Edite Casé de Oliveira \\ ORCID: https://orcid.org/0000-0003-2266-5890 \\ Universidade Federal da Paraíba, Brasil \\ E-mail: milacdo1@gmail.com \\ Kedma Anne Lima Gomes \\ ORCID: https://orcid.org/0000-0001-6720-011X \\ Universidade Federal da Paraíba, Brasil \\ E-mail: kalg2010@hotmail.com \\ Waleska Fernanda Souto Nóbrega \\ ORCID: https://orcid.org/0000-0001-8140-4063 \\ Universidade Estadual da Paraíba, Brasil \\ E-mail: drawaleskasouto@gmail.com \\ Gustavo Correia Basto da Silva \\ ORCID: https://orcid.org/0000-0002-6081-2540 \\ Universidade Estadual da Paraíba, Brasil \\ E-mail: gugacorreiaa@gmail.com \\ Danilo Vieira Barbosa \\ ORCID: https://orcid.org/0000-0003-0386-8895 \\ Universidade Federal de Campina Grande, Brasil \\ E-mail: danilo_vieira23@hotmail.com \\ Ramonyele Gomes Franklin \\ ORCID: https://orcid.org/0000-0001-5723-5693 \\ Universidade Estadual da Paraíba, Brasil \\ E-mail: nyele.gomes@gmail.com \\ Rayane Dantas dos Santos \\ ORCID: https://orcid.org/0000-0001-5512-851X \\ Universidade Estadual da Paraíba, Brasil \\ E-mail: dantas.rayane@gmail.com
}

\begin{abstract}
Resumo
O câncer de mama é considerado o câncer mais incidente em mulheres no mundo. Evidências tem mostrado que os canabinoides podem modular vias de sinalização responsáveis pelo crescimento e para a propagação do câncer. Dessa forma, a presente revisão da literatura buscou sumarizar as evidências existentes até então, acerca do uso da cannabis para o tratamento do câncer de mama. A coleta de dados foi realizada no período de maio a junho de 2021, na base de dados eletrônica PubMed. Foram incluídos artigos desde sua origem até 01 de junho de 2021, com a combinação dos seguintes descritores: ((Cannabidiol OR cannabis) AND "breast neoplasms"). Os resultados demonstraram a escassez de estudos que buscaram avaliar os efeitos da cannabis medicinais no tratamento do câncer de mama em humanos. Como conclusão, o estudo permitiu compreender a importância que a cannabis medicinal, em especial, o cannabidiol, vem fornecendo para os avanços no tratamento antineoplásico em células in vitro.
\end{abstract}

Palavras-chave: Cannabis; Cannabidiol; Câncer de mama; Tratamento antineoplásico.

\begin{abstract}
Breast cancer is considered the most incident cancer in women worldwide. Evidence has shown that cannabinoids can modulate signaling pathways responsible for cancer growth and spread. Thus, this literature review sought to summarize the evidence that existed so far on the use of cannabis for the treatment of breast cancer. Data collection was carried out from May to June 2021, in the electronic database PubMed. Articles from their origin to June 1, 2021 were included, with the combination of the following descriptors: ((Cannabidiol OR cannabis) AND "breast neoplasms"). The results demonstrated the paucity of studies that sought to assess the effects of medicinal cannabis in the treatment of breast cancer in humans. In conclusion, the study allowed us to understand the importance that medicinal cannabis, in particular cannabidiol, has been providing for advances in anticancer treatment in cells in vitro. Keywords: Cannabis; Cannabidiol; Breast câncer; Anticancer treatment.
\end{abstract}

\section{Resumen}

El cáncer de mama se considera el cáncer más común en mujeres en todo el mundo. La evidencia ha demostrado que los cannabinoides pueden modular las vías de señalización responsables del crecimiento y la propagación del cáncer. 
Por lo tanto, esta revisión de la literatura buscó resumir la evidencia que existía hasta ahora sobre el uso de cannabis para el tratamiento del cáncer de mama. La recolección de datos se realizó de mayo a junio de 2021, en la base de datos electrónica PubMed. Se incluyeron artículos desde su origen hasta el 1 de junio de 2021, con la combinación de los siguientes descriptores: ((Cannabidiol O cannabis) Y "neoplasias de mama"). Los resultados demostraron la escasez de estudios que buscaban evaluar los efectos del cannabis medicinal en el tratamiento del cáncer de mama en humanos. En conclusión, el estudio permitió comprender la importancia que el cannabis medicinal, en particular el cannabidiol, viene aportando a los avances en el tratamiento anticáncer en células in vitro.

Palabras clave: Cannabis; Cannabidiol; Cáncer de mama; Tratamiento contra el cáncer.

\section{Introdução}

O câncer de mama $(\mathrm{CM})$ é considerado o câncer mais incidente em mulheres no mundo. Em 2018, o mesmo apresentou um risco estimado de 55,2/100 mil (Ferlay et al., 2018). De acordo com Instituto Nacional do Câncer (INCA), a estimativa de câncer de mama para o Brasil é de 66.280 casos novos para cada ano do triênio 2020-2022, correspondendo a um risco estimado de 61,61 novos casos a cada 100 mil mulheres (INCA, 2019).

O CM classifica-se em não metastático e metastático e seus principais tratamentos consistem em: i) terapias locais: caracterizadas pela cirurgia e pela radioterapia e ii) terapias sistêmicas: subdivididas em hormonioterapia e quimioterapia (American Cancer Society, 2019; Waks \& Winer, 2019). No entanto, as toxicidades relacionadas ao tratamento quimioterápico e seus efeitos adversos ainda se apresentam como um problema para a comunidade científica (Anderson, Holstein \& Kedar, 2020).

A Cannabis vem sendo cultivada e utilizada de forma recreativa e terapêutica há séculos por diferentes culturas e recentemente, esta foi retirada da lista de substâncias proscritas, o que fez o Conselho Federal de Medicina autorizar seu uso com terapêutica alternativa para aqueles pacientes que não respondem aos tratamentos convencionais em diversos tipos de patologias (Oliveira \& Lima, 2016).

A literatura tem demonstrado que o uso de canabinoides tem apresentado importantes benefícios no tratamento de diversas doenças, em especial no alívio da dor crônica, na redução de sintomas de déficit de atenção, hiperatividade e transtorno pós-traumático, tendo se mostrado como um possível substituto para os tratamentos tradicionais que utilizam opioides, antidepressivos e benzodiazepínicos (Rojas - Raras et al., 2019).

Para além disso, evidências tem mostrado que os canabinoides podem modular vias de sinalização responsáveis pelo crescimento e para a propagação do câncer. Isso ocorre porque eles são capazes de inibir a progressão do ciclo celular e as quimiotaxias, bloqueando a angiogênese (Alexander, Smith, \& Rosengren, 2009). Além disso, estudos recentes têm apresentado relação entre os canabinóides e a indução de células autofágicas à morte (Salazar et al., 2009).

O sistema endocanabinoide compreende dois principais receptores endocamabinóides: (i) CB1, encontrado especialmente no sistema nervoso; e (ii) CB2, expresso no sistema periférico e no cérebro. Ele tem sido muito estudado por seu envolvimento no desenvolvimento do câncer, incluindo características, tais quais: angiogênese, apoptose e metástases (Marzo \& Matias, 2005).

A neuroquímica ocorre por meio do canabidiol (CBD), substância presente na cannabis sativa, pertence à família dos canabinóides, que apresenta um grupo de compostos farmacologicamente ativos que se ligam com receptores acoplados à proteína G (Pertwee \& Ross, 2002). A esse respeito, uma pesquisa recente apresentou que o CBD inibiu o crescimento do CM e diminui o regulador de metástase em células de câncer de mama (Ligresti, 2006).

Apesar do avanço nas pesquisas existentes na área e das evidências que em sido apresentadas em relação ao uso de substâncias da cannabis para o tratamento do CM ainda é um assunto que apresenta um certo tabu, em recorrência da criminalização da mesma, especialmente no Brasil. Dessa forma, a presente pesquisa buscou sumarizar as evidências existentes até então, acerca do uso da cannabis para o tratamento do câncer de mama. 


\section{Metodologia}

Tratou-se de uma revisão integrativa da literatura que teve por objetivo analisar os artigos existentes na literatura acerca do uso da cannabis para o tratamento do câncer de mama. A coleta de dados foi realizada no período de maio a junho de 2021, na base de dados eletrônica PubMed.

A revisão integrativa da literatura consiste em um método de pesquisa que visa a busca, a avaliação crítica e a síntese das evidências disponíveis sobre determinado tema, cujo produto final engloba o estado atual do conhecimento do assunto investigado, que pode servir de subsídio para a implementação de intervenções efetivas na assistência à saúde (Mendes, Silveira \& Galvão, 2008).

Foram incluídos artigos desde sua origem até 01 de junho de 2021, com a combinação dos seguintes descritores: ((Cannabidiol OR cannabis) AND “breast neoplasms”). Os descritores selecionados eram controlados pelo MeSH. Os critérios de inclusão considerados foram: (i) estudos empíricos, (ii) língua inglesa, (ii) que buscaram analisar o uso da cannabis durante o tratamento do câncer de mama. O critério de exclusão foi: (i) estudos realizados com modelos animais, ou (ii) estudos in vitro.

Inicialmente, após a inclusão da estratégia de busca na base de dados selecionada, foram apresentados 39 resultados. Estes foram submetidos a leitura dos títulos e resumos para triagem dos artigos que remetiam ao tema de interesse. Posteriormente, os artigos restantes foram lidos na íntegra para aplicação dos critérios de elegibilidade. A estratégia de busca pode ser visualizada na Figura 1. Ao final, restaram 13 artigos para síntese qualitativa, que permite uma compreensão ampla do fenômeno em estudo (Souza, Marques-Vieira, Severino \& Antunes, 2017).

Figura 1 - Fluxograma dos artigos selecionados para Revisão de Literatura

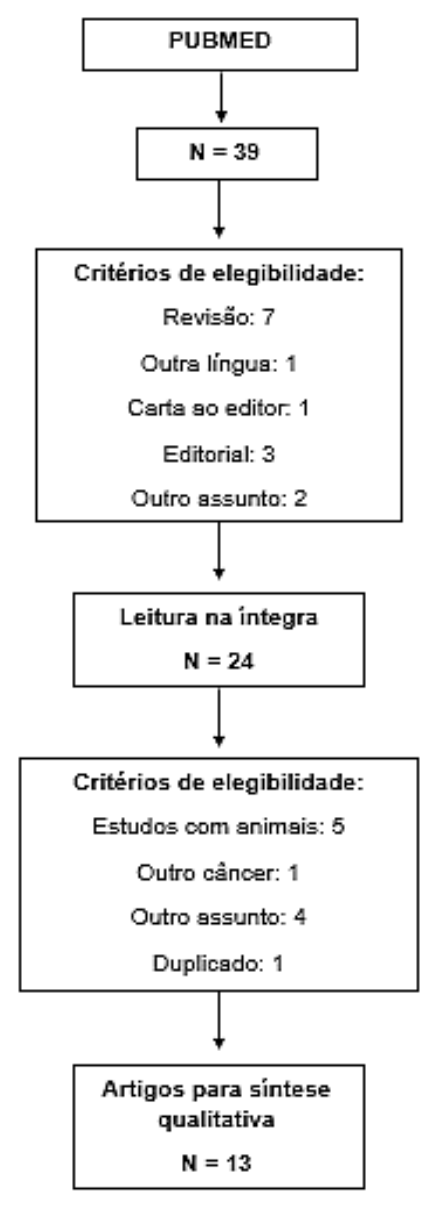

Fonte: Dados da pesquisa (2021). 


\section{Resultados}

O resultado de busca apresentou 13 artigos para síntese qualitativa. Foram incluídos artigos de 1997 a 2020. Todos eles buscaram avaliar substâncias da cannabis em células humanas cancerígenas.

Shrivastava et al. (2011) realizaram a cultura de células humanas (MDA-MB-231, MCF-7, SK-BR-3, ZR-75-1 e MCF-10A) para avaliar se o CBD induz a morte celular programada em células com câncer de mama. Os resultados apresentaram sugeriram que o CBD induziu apoptose e autofagia nas células cancerígenas. Além disso, autores tem relatado os efeitos do CBD na interação entre a apoptose e a aufagia, apresentando uma melhor precisão na maneira como essa morte celular é induzida, auxiliando no processo de definição da aplicação ideal do CBD para a terapêutica do câncer (Schoeman, Beukes \& Frost, 2020; Shrivastava et al., 2011, Takeda et al., 2012).

O estudo realizado por Suzuki et al. (2017), buscou avaliar os efeitos do ácido canabidiólico (CBDa) na atividade transcricional do completo de proteína-1 (AP-1), considerado um regulador dos genes envolvidos na proliferação, transformação e morte celular. Para isso eles isolaram folhas de cannabis (pureza > 98\%) e uma amostra de CBD disponível comercialmente (pureza $=96,5 \%$ ). Todos os outros reagentes foram utilizados sem purificação adicional. Foi realizada a cultura de células humanas cancerígenas (MDA-MB-231) em vermelho de fenol. Os resultados encontrados ressaltaram pela primeira vez que CBD exerceu efeitos inibitórios na atividade transcricional mediada por AP-1 em um modo dependente da concentração.

A esse respeito, estudos realizados com células in vitro apresentou a CBD como um potencial fator para supressão de genes envolvidos na metástase do câncer (McAllister et al., 2011, Takeda et al., 2014). As evidências apresentam ainda, que a CBD é um importante regulador na expressão do COX-2, e que isso ocorre por meio da sinalização relacionado ao PPAR $\beta / \delta$, sendo considerado um importante fator medicinal (Elbaz et al., 2015, Hirao-Suzuki et al., 2019, Takeda et al., 2016).

Blasco-Benito et al. (2018) compararam a eficácia antitumoral do $\Delta 9$-tetrahidrocanabinol (THC) puro com uma preparação botânica da cannabis (BDP), produzida pela combinação de diversos composto da planta. Os resultados apresentaram que o BDP forneceu melhores resultados na produção de respostas antitumorais em cultura de células e em modelos animais para todos os tipos de câncer quando comparado ao THC puro. Isso se deu, em parte, ao efeito de ação dos dois mecanismos. Enquanto o THC ativou os receptores de CBD, o BDP modulou diferentes mecanismos de ação. Assim, o estudo sugere que a preparação de drogas considerando os diversos compostos da cannabis pode ser mais eficaz para o controle do câncer de mama do que a utilização do CBD puro (Blasco-Benito et al., 2018).

Sultan, Marie \& Sheweita (2018) avaliaram o efeito do CBD em duas diferentes linhas celulares de CM (ER-positivo e triplo negativo). O CBD induziu a apoptose e inibiu a sobrevivência celular em ambos os tipos de câncer. Os autores sugerem que isso ocorreu porque o CBD induziu a interação entre a proteína PPARy, o mTOR e ciclina D1, e que esse mecanismo auxiliou na apoptose celular. Dessa forma, o estudo considera que o CBD pode ser considerado um importante tratamento para diferentes subtipos neoplásicos.

Um estudo buscou avaliar o potencial de atividade antitumoral do aminoalquililindol, considerando sua interação com terapias convencionais, como a radioterapia. As evidências sugeriram um potencial nos compostos de aminoalquilindol no aumento da eficácia do tratamento por radiação no câncer de mama. Além disso, o estudo apresentou que outros compostos, como o THC e a nabilona não interferiram na eficácia da terapia de radiação (Sean et al., 2013). Nessa perspectiva, o estudo realizado por Ruh et al. (1997) propôs que compostos psicoativos ou inativos da classe estrutural canabinoide (THC) não se apresentaram como agonistas nas respostas do receptor de estrogênio em ensaios com células humanas cancerígenas in vitro. 


\section{Resultados}

O objetivo do artigo consistiu em sumarizar as evidências apresentadas acerca dos efeitos das substâncias presentes na cannabis para o tratamento antineoplásico em células in vitro. Percebeu-se um avanço na compreensão das bases bioquímicas envolvidas nesse processo (Blasco-Benito et al., 2018, Sultan, Marie \& Sheweita, 2018; Sean et al., 2013).

No tocante à eficácia que o CBD apresentou na inibição da metástase tumoral (Elbaz et al., 2015), é importante considerar que o desenvolvimento de metástases tem sido considerado um dos principais indicadores do aumento da mortalidade em pacientes oncológicos (Jin et al., 2014). Dessa forma, considera-se que o CBD vem apresentando resultados promissores do avanço do tratamento contra o câncer.

Como apresentado anteriormente, o estudo de Blasco-Benito et al. (2018) avaliou o composto de diferentes substâncias da cannabis no tratamento antineoplásico, apresentando avanços significativos para a pesquisa na área. A esse respeito, apesar da nossa medicina atual ter apostado em compostos puros para o tratamento do câncer (a exemplo do THC), é importante considerar que uma doença tão complexa pode exigir abordagens multidimensionais, permitindo o combate simultâneo de diferentes degradações causadas pelo câncer. Nesse interim, a interação de diferentes compostos da cannabis já foi relatado em estudos anteriores (Nadal et al., 2017; Ben-Shabat et al., 1998).

Embora os nossos resultados tenham apresentado uma importância considerável das substâncias da cannabis no tratamento oncológico, percebe-se que a farmacologia dessas drogas é altamente complexa, especialmente ao considerar que a utilização dessas substâncias de forma simultânea pode produzir resultados mais eficazes do que a substância pura.

O presente estudo apresenta algumas limitações, entre elas, a coleta dos artigos em apenas uma base pode ter prejudicado o alcance das pesquisas. Assim, sugere-se a realização de revisões mais abrangentes, de modo a propiciar uma melhor análise dos avanços existentes até o momento relacionados à questão da pesquisa.

Salienta-se ainda que a presente revisão buscou analisar apenas estudos in vitro. Alguns estudos in vivo já foram publicados na literatura e tem demonstrado potencial terapêutico promissor, no entanto, devido às limitações para sua realização e às metodologias diversificadas que utilizam, torna-se necessária ainda a realização de novos estudos que preencham as lacunas ainda existentes no que diz respeito a investigação dos efeitos benéficos da cannabis, essencialmente no que diz respeito ao câncer e a sua influência no tratamento antineoplásico (Viera, Marques \& Sousa, 2020).

\section{Considerações Finais}

Como conclusão, o estudo permitiu compreender a importância que a cannabis medicinal, em especial, o cannabidiol, vem fornecendo para os avanços no tratamento antineoplásico em células in vitro. No entanto, a busca realizada refletiu a escassez de estudos que buscaram avaliar os efeitos da cannabis medicinais no tratamento do câncer de mama em humanos. Apesar dos estudos com células in vitro terem apresentado resultados positivos, estes podem não ser sustentados na complexidade humana. Por isso, a necessidade em investigar tal hipótese, afim de fornecer uma melhor compreensão desse mecanismo.

\section{Referências}

Alexander, A., Smith, P. F., \& Rosengren, R. J. (2009). Cannabinoids in the treatment of cancer. Cancer Lett, 285 , 6-12.

American Cancer Society. (2019). Hormone Therapy for Breast Cancer. Disponível em: https://www.cancer.org/cancer/breast-cancer/treatment/hormonetherapy-for-breast-cancer.html. Acessado em: 08 de setembro de 2020. Anderson, D.E., Holstein, S.A., \& Kedar, S. (2020). Visual Pathway Degeneration in Chemotherapy-Related Neurotoxicity: A Review and Directions for Future Research. Neuro-Ophthalmology, 1-9. Doi:10.1080/01658107.2019.1702703.

Ben-Shabat, S., Fride, E., Sheskin, T., Tamiri, T., Rhee, M.H., Vogel, Z., Bisogno, T., De Petrocellis, L., Di Marzo, V., \& Mechoulam, R. (1998). An entourage effect: inactive endogenous fatty acid glycerol esters enhance 2-arachidonoyl-glycerol cannabinoid activity. Eur J Pharmacol, 353(1), 23-31. 
Research, Society and Development, v. 10, n. 10, e09101018387, 2021

(CC BY 4.0) | ISSN 2525-3409 | DOI: http://dx.doi.org/10.33448/rsd-v10i10.18387

Ferlay, J., Ervik, M., Lam, F., Colombet, M., Mery, L., Piñeros, M., Znaor, A., Soerjomataram, I., \& Bray, F. (2018). Cancer Today (powered by GLOBOCAN 2018). Disponível em: https://publications.iarc.fr/Databases/Iarc-Cancerbases/Cancer-Today-Powered-By-GLOBOCAN-2018--2018. Acessado em: 04 julho 2021.

INCA. (2019). Estimativa 2020: Incidência do Câncer no Brasil. Rio de Janeiro: INCA.

Jin, U. H., Lee, S. O., Pfent, C., \& Safe, S., (2014). The aryl hydrocarbon receptor ligand omeprazole inhibits breast cancer cell invasion and metastasis. BMC Cancer, 14, 498.

Ligresti, A., Moriello, A.S., Starowicz, K., Matias, I., Pisanti, S., De Petrocellis, L., Laezza, C., Portella, G., Bifulco, M., \& Di Marzo, V. (2006). Antitumor activity of plant cannabinoids with emphasis on the effect of cannabidiol on human breast carcinoma. J Pharmacol Exp Ther, 318, $1375-1387$.

Marzo, V., \& Matias, I. (2005). Endocannabinoid control of food intake and energy balance. Nat Neurosci, 8, 585-589.

Mendes, K. D. S., Silveira, R. C. P. S. \& Galvão, C. M. Revisão integrativa: método de pesquisa para a incorporação de evidências na saúde e na enfermagem. Texto e Contexto Enfermagem. 17(4), 2008.

Nadal, X., Del Rio, C., Casano, S., Palomares, B., Ferreiro-Vera, C., Navarrete, C., Sanchez-Carnerero, C., Cantarero, I., Bellido, M. L., Meyer, S., Morello, G., Appendino, G., \& Muñoz, E. (2017). Tetrahydrocannabinolic acid is a potent PPARgamma agonist with neuroprotective activity. Br J Pharmacol, 174 (23), 4263-4276. DOI: $10.1111 /$ bph.14019.

Oliveira, K. L. B., Lima, T. P. S. Cannabis Sativa: Potencial Terapêutico. [Trabalho de Conclusão de Curso]. Bacharelado em Biomedicina. Faculdade São Lucas: Porto Velho - RO, 30p.

Pertwee, R. G., \& Ross, R. A. (2002). Cannabinoid receptors and their ligands. Prostaglandins Leukot Essent Fatty Acids, 66, 101-121.

Rojas - Raras, C., Polanco-Carrasco, R. Cisterna, A., Hernandez, V., Miranda, F., Moreno, A., Alarcon, L. Uso medicinal de cannabis: una revisión de la evidencia. Tarapia Psicológica, 37(2), 2019.

Salazar, M., Carracedo, A., Salanueva, I.J., Hernandez-Tiedra, S., Lorente, M., Egia, A., Vásquez, P., Blázquez, C., Torres, S., García, S., Nowak, J., Fimia, G. M., Piacentini, M., Cecconi, F., Pandolfi, P. P., González-Feria, L., Lovanna, J. L., Guzmán, M., Boya, P., \& Velasco, G. (2009). Cannabinoid action induces autophagy-mediated cell death through stimulation of ER stress in human glioma cells. J Clin Invest, 119, 1359-1372.

Souza, L. M. M., Marques-Vieira, C. M. A., Severino, S. S. P. \& Antunes, A. V. A metodologia de revisão integrativa da literatura em Enfermagem. Revista Investigação em Enfermagem. 21(2), 2017.

Vieira, L. S., Marques, A. E. F., Sousa, V. A. O uso de Cannabis sativa para fins terapêuticos no Brasil: uma revisão de literatura. Scientia Naturalis, 2(2), 901919,2020

Waks, A. G., \& Winer, E. P. (2019). Breast Cancer Treatment: A Review. JAMA, 321(3):288-300. Doi: 10.1001/jama.2018.19323. 